\title{
Pre-evaluation Strategy on Algorithms for Mining Top - k High Utility Item Sets
}

\author{
M. V. Mali \\ Department of Computer Science and \\ Engineering, V.V.P. Institute of Engineering and \\ Technology, \\ Solapur, Maharashtra, India -413004
}

\author{
H. B. Torvi \\ Assistant Professor, Department of Computer \\ Science and Engineering, V.V.P. Institute of \\ Engineering and Technology, Solapur, \\ Maharashtra, India -413004
}

\begin{abstract}
A rising trend in data mining is a High utility item sets (HUIs) mining. It aims to find all item sets which have an utility which meets a client determined least utility edge min_util. But, for clients, it is an issue to set a min_util efficiently. So, it is not proper procedure for clients to find a least utility edge by experimentation. An excessive number of HUIs will be produced, in the case that min_util is set very low. Due to this the mining procedure may result wasteful. It is also possible that no HUIs be found, if min_util is set very high. So for addressing the above issues, we redefine the problem of high utility item sets (HUIs) mining by top-k high utility item sets ( top-k HUI ) mining. Here, desired number of HUIs to be mined is $\mathrm{k}$. Two different algorithms which are named as TKU and TKO (mining Top-K Utility item sets in two stages, mining Top-K utility item sets in one stage, respectively) are proposed for mining the item sets without setting the value of min_util. We apply pre-evaluation strategy to algorithms to improve the performance.
\end{abstract}

\section{General Terms}

Data mining ,High utility mining

\section{Keywords}

Utility mining, high utility item set mining, top-k high utility item set mining, frequent item set, transactional database.

\section{INTRODUCTION}

A fundamental research topic in data mining is Frequent item set mining (FIM). But, a large amount of frequent but lowvalue item sets are discovered in the traditional FIM and lose the information on valuable item sets which may have low selling frequencies. Hence, it can't satisfy the requirement of users who desire to discover item sets with high utilities such as high profits. An item set is said to be frequent if its support is no less than a given minimum support threshold. Hundreds of studies have been conducted on this topic. However, an important limitation of FIM is its assumptions that all items have the same importance to the user (e.g. unit profit or weight) and that items may not appear(quantity) more than once in each transaction.

These assumptions often do not hold in real life. For example, in transaction databases, items may have different unit profits, and items in transactions may be associated with different purchase quantities. Besides, in real-life applications, retailers may be more interested in finding item sets that yield a high profit rather than discovering frequent item sets.

Utility mining is nothing but the discovery of item sets with utilities higher than a user-specified minimum utility threshold. It has a wide range of applications including ecommerce. But a difficult problem here is to set an appropriate minimum utility threshold. Many ( too much) high utility item sets will be generated if in case, the minimum threshold is set too low. Also it may take a long time to compute. On the other hand setting the minimum threshold too high may result in too few results. Setting appropriate minimum utility threshold by trial and error is not very efficient. To precisely control the output size and discover the item sets with the highest utilities without setting the thresholds, a promising solution is to redefine the task of mining HUIs as mining top-k high utility item sets (top-k HUIs). The idea is to let the users specify $k$, i.e., the number of desired item sets, instead of specifying the minimum utility threshold. Setting $\mathrm{k}$ is more comfortable than setting the threshold because $\mathrm{k}$ represents the number of item sets that the users want to find.

An efficient algorithm named TKU (Top-K Utility item sets mining) and TKO (mining Top-K utility item sets in one stage) are proposed for mining such item sets without the need to set min_util.

\section{LITERATURE REVIEW}

In this section, studies related to high utility item set mining, top-k frequent pattern mining and top $-\mathrm{k}$ high utility pattern mining are briefly reviewed.

\subsection{High Utility Itemset Mining}

High utility item set mining[3] has received lots of attention in recent years. Also many efficient algorithms have been proposed. Some of them are HUI-Miner, Two-Phase, IIDS, IHUP, UPGrowth and d2HUP. These algorithms can be generally categorized into two types: two phase and one-phase algorithms.

The main characteristic of two-phase algorithms is that they consist of two phases.

Phase one generates a set of candidates that are potential high utility item sets. And the second phase calculates the exact utility of each candidate found in the first phase to identify high utility item sets.

Unlike this, the discovery of high utility item sets using only one phase and the production of no candidates are the main characteristics of one-phase algorithms. d2HUP and HUIMiner are one-phase algorithms. Even if above studies may perform well in some applications, they are not developed for top-k high utility itemset mining. Also it suffers from the problem of setting appropriate thresholds.

\subsection{Top-k Frequent Pattern Mining}

Many studies have been proposed to mine different kinds of top-k patterns[8], such as top-k frequent item sets, top-k frequent closed item sets, top-k closed sequential patterns, 
top-k association rules, top-k sequential rules, top-k correlation patterns.

\subsection{Top - k High Utility Pattern Mining}

Chan et al. introduced the task of top-k high utility pattern mining[11] . But the definition of high utility itemset used in this project and in their study is different. Chan et al.'s study has considered utilities of various items, but they have not considered the quantitative values of items in transactions. In this project, we are defining the task of top-k high utility itemset mining by considering both quantities and profits of items.

\subsection{Top - k High Utility Itemset Mining}

We try to solve above issues by redefining the problem of high utility item sets (HUIs) mining by top-k high utility item sets ( top-k HUI) mining[1]. Here $\mathrm{k}$ is the desired number of HUIs to be mined. For this, two algorithms are proposed for mining such item sets without the need to set min_util.

\section{The TKU Algorithm:}

The baseline approach TKU is an extension of UPGrowth , a tree-based algorithm for mining HUIs. TKU adopts the UPTree structure of UP-Growth to maintain the information of transactions and top-k HUIs. TKU is executed in three steps:

(1) Constructing the UP-Tree.

(2) Generating potential top-k high utility itemsets (PKHUIs) from the UP-Tree.

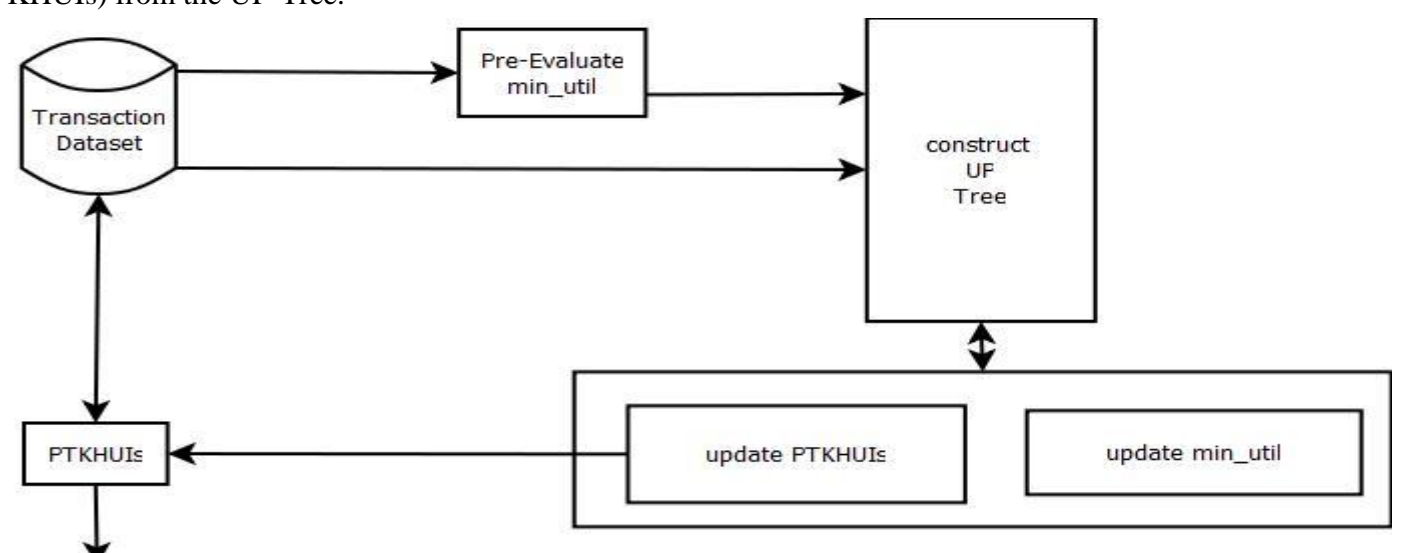

Top-K HU]

Fig 1: System design for TKU with Pre-Evaluation

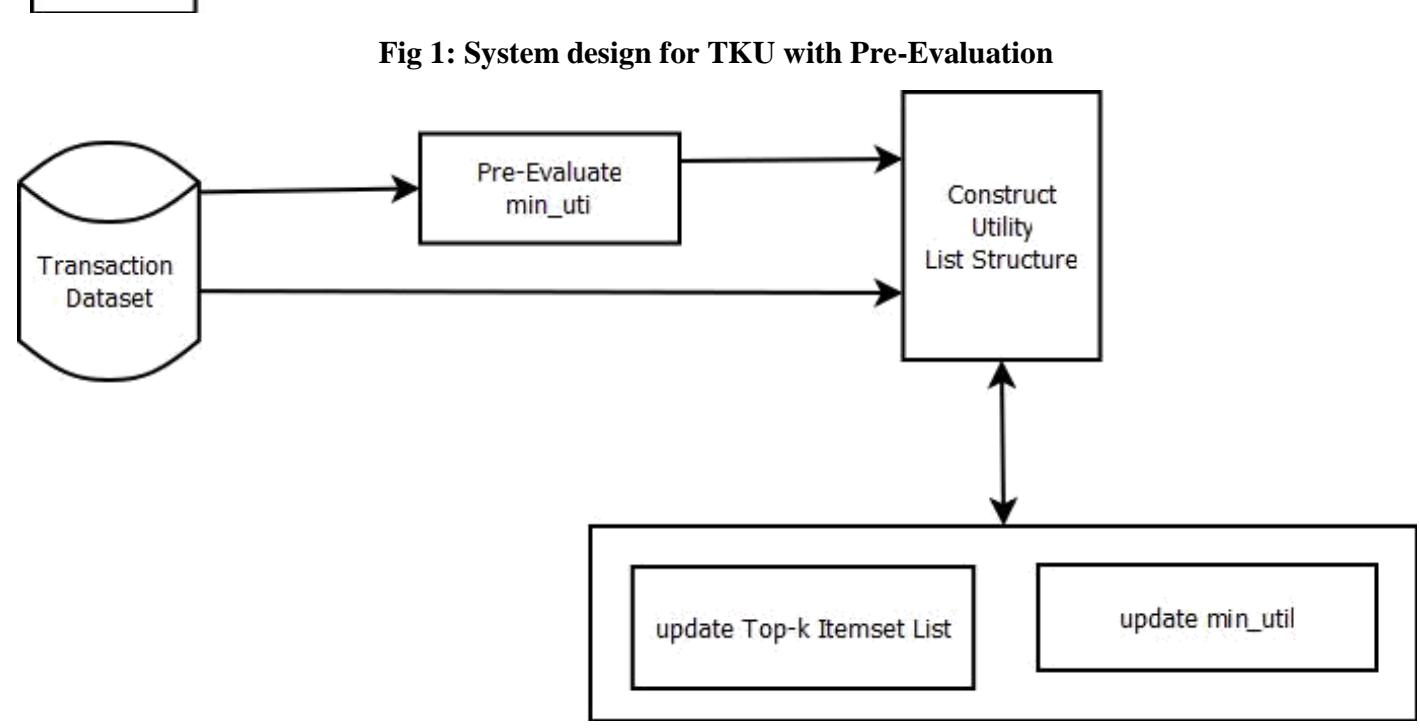

Fig 2: System design for TKO with Pre-Evaluation
The TKO Algorithm:

The TKO (mining Top-k utility itemsets in One phase) can discover top-k HUIs in only one phase. It utilizes the basic search procedure of HUI-Miner and its utility-list structure . Whenever an itemset is generated by TKO, its utility is calculated by its utility-list without scanning the original database.

\section{PROPOSED SYSTEM}

In proposed system, for better performance of both algorithms TKU and TKO, we will apply Pre-evaluation strategy to them.

\section{Pre-Evaluation Strategy of Min_Util :}

Though TKU provides a way to mine top-k HUIs, min_util is set to 0 before the construction of the UP-Tree. This results in the construction of a full UP-Tree in memory, which degrades the performance of the mining task. The number of nodes maintained in memory could be reduced if min_util could be raised before the construction of the UP-Tree and more unpromising items in transactions sets could be pruned more unpromising items in transactions, and the mining algorithm could achieve better performance. Based on this idea, we propose a strategy named PE (Pre-evaluation Step) to raise min_util during the first scan of the database. 


\section{METHODOLOGY}

In the proposed system, in advance, we are calculating the min_util before the algorithms named TKU (mining Top-K Utility item sets in two stages) and TKO (mining Top-K utility item sets in one stage) starts working on transactions . Calculation of the min_util is done by the Pre-evaluation matrix method which considers the transactions, items and their utility. According to this strategy, min_util could be raised before the construction of the UP-Tree and prune more unpromising items in transactions, the number of nodes maintained in memory could be reduced and the mining algorithm could achieve better performance, while finding top k HUIs.

\section{EXPERIMENTAL RESULTS}

Following tables shows the time required for finding top-k HUIs by TKU and TKO with and without pre-evaluation of min_util. Two different datasets are used.

\subsection{Supermarket Dataset} a)TKU

Table 1. Supermarket Dataset TKU

\begin{tabular}{|c|c|c|c|c|}
\hline K & $\begin{array}{c}\text { min_util } \\
\text { Zero }\end{array}$ & $\begin{array}{c}\text { min_util } \\
\text { nonzero }\end{array}$ & $\begin{array}{c}\text { \% Improvement } \\
\text { in Performance }\end{array}$ & Min_util \\
\hline 1 & 2356 & 1885 & 19.99 & 2103 \\
\hline 10 & 2401 & 1777 & 25.98 & 2159 \\
\hline 20 & 2525 & 1818 & 28.00 & 2203 \\
\hline 30 & 2547 & 1885 & 25.99 & 2249 \\
\hline 40 & 3015 & 2300 & 23.71 & 2305 \\
\hline 50 & 3079 & 2433 & 20.98 & 2315 \\
\hline 60 & 3120 & 2496 & 20.00 & 2326 \\
\hline 70 & 3157 & 2337 & 25.97 & 2359 \\
\hline 80 & 3162 & 2372 & 24.98 & 2378 \\
\hline 90 & 3429 & 2504 & 26.97 & 2415 \\
\hline
\end{tabular}

\section{b)TKO}

Table 2. Supermarket Dataset TKO

\begin{tabular}{|c|c|c|c|c|}
\hline K & $\begin{array}{c}\text { min_util } \\
\text { Zero }\end{array}$ & $\begin{array}{c}\text { min_util } \\
\text { nonzero }\end{array}$ & $\begin{array}{c}\text { \% Improvement } \\
\text { in Performance }\end{array}$ & Min_util \\
\hline 1 & 2215 & 1716 & 22.52 & 2103 \\
\hline 10 & 2233 & 1635 & 26.78 & 2159 \\
\hline 20 & 2324 & 1673 & 27.98 & 2203 \\
\hline 30 & 2344 & 1772 & 24.40 & 2249 \\
\hline 40 & 2800 & 2115 & 24.46 & 2305 \\
\hline 50 & 2895 & 2300 & 20.55 & 2315 \\
\hline 60 & 2871 & 2272 & 20.86 & 2326 \\
\hline 70 & 2968 & 2127 & 28.33 & 2359 \\
\hline 80 & 3004 & 2159 & 28.12 & 2378 \\
\hline 90 & 3155 & 2304 & 26.97 & 2415 \\
\hline
\end{tabular}

\subsection{Stationary Shop Dataset a) TKU}

Table 3. Stationary Datasett TKU

\begin{tabular}{|c|c|c|c|c|}
\hline K & $\begin{array}{c}\text { min_util } \\
\text { Zero }\end{array}$ & $\begin{array}{c}\text { min_util } \\
\text { nonzero }\end{array}$ & $\begin{array}{c}\text { \% Improvement } \\
\text { in Performance }\end{array}$ & Min_util \\
\hline 1 & 2348 & 1855 & 20.99 & 1903 \\
\hline 10 & 2401 & 1777 & 25.98 & 1959 \\
\hline 20 & 2525 & 1818 & 28.00 & 2003 \\
\hline 30 & 2547 & 1885 & 25.99 & 2015 \\
\hline 40 & 3015 & 2300 & 23.71 & 2205 \\
\hline 50 & 3079 & 2433 & 20.98 & 2295 \\
\hline 60 & 3120 & 2496 & 20.00 & 2298 \\
\hline 70 & 3157 & 2337 & 25.97 & 2359 \\
\hline 80 & 3162 & 2372 & 24.98 & 2389 \\
\hline 90 & 3429 & 2504 & 26.97 & 2412 \\
\hline
\end{tabular}

b)TKO

Table 4. Stationary Datasett TKO

\begin{tabular}{|c|c|c|c|c|}
\hline K & $\begin{array}{c}\text { min_util } \\
\text { Zero }\end{array}$ & $\begin{array}{c}\text { min_util } \\
\text { nonzero }\end{array}$ & $\begin{array}{c}\text { \% Improvement } \\
\text { in Performance }\end{array}$ & Min_util \\
\hline 1 & 2195 & 1759 & 19.86 & 1903 \\
\hline 10 & 2233 & 1635 & 26.78 & 1959 \\
\hline 20 & 2324 & 1673 & 28.01 & 2003 \\
\hline 30 & 2344 & 1772 & 24.40 & 2015 \\
\hline 40 & 2800 & 2115 & 24.46 & 2205 \\
\hline 50 & 2895 & 2300 & 20.55 & 2295 \\
\hline 60 & 2871 & 2272 & 20.86 & 2298 \\
\hline 70 & 2968 & 2127 & 28.33 & 2359 \\
\hline 80 & 3004 & 2159 & 28.12 & 2389 \\
\hline 90 & 3155 & 2304 & 26.97 & 2412 \\
\hline
\end{tabular}

\section{CONCLUSION}

In the proposed system, an attempt is made to improve the performance of algorithms named TKU (mining Top-K Utility item sets in two stages) and TKO (mining Top-K utility item sets in one stage) by the use of pre-evaluation strategy. According to this strategy, min_util is calculated and raised before the construction of the UP-Tree and pruned more unpromising items in transactions. Also, the number of nodes maintained in memory is reduced and the mining algorithm achieves better performance. In future, for better performance, while calculating min_util, instead of considering all items, , the items with the less profit can be neglected. 


\section{REFERENCES}

[1] V. Kavitha, B. G. Geetha, "Review on high utility itemset mining algorithms" IEEE Trans. Knowl. Data Eng., vol. 21, no. 12, pp. 1708-1721, October 2016.

[2] Song Wei, Liu Yu, Li Jinhong, "Mining high utility itemsets by dynamically pruning the tree structure", Applied intelligence, vol. 40, no. 1, pp. 29-43, 2014.

[3] Vincent S. Tseng et al., "Efficient Algorithms for Mining the Concise and Lossless Representation of High Utility Itemsets", Knowledge and Data Engineering IEEE Transactions on, vol. 27, no. 3, pp. 726-739, 2015.

[4] C. Ahmed, S. Tanbeer, B. Jeong, and Y. Lee, "Efficient tree structures for high-utility pattern mining in incremental databases," IEEE Trans. Knowl. Data Eng., vol. 21, no. 12, pp. 1708-1721, Dec. 2009.

[5] K. Chuang, J. Huang, and M. Chen, "Mining top-k frequent patterns in the presence of the memory constraint," VLDB J., vol. 17, pp. 1321-1344, 2008.
[6] R. Chan, Q. Yang, and Y. Shen, "Mining high-utility itemsets," in Proc. IEEE Int. Conf. Data Mining, 2003, pp. 19-26.

[7] M. Liu and J. Qu, "Mining high utility itemsets without candidate generation," in Proc.ACM Int. Conf. Inf. Knowl. Manag., 2012, pp. 55-64.

[8] P. Fournier-Viger, C.Wu, and V. S. Tseng, "Mining topk association rules," in Proc. Int. Conf. Can. Conf. Adv. Artif. Intell., 2012, pp. 61-73.

[9] P. Fournier-Viger, C. Wu, and V. S. Tseng, "Novel concise representations of high utility itemsets using generator patterns," in Proc. Int. Conf. Adv. Data Mining Appl. Lecture Notes Comput. Sci., 2014, vol. 8933, pp. $30-43$.

[10] J. Han, J. Pei, and Y. Yin, "Mining frequent patterns without candidate generation," in Proc. ACM SIGMOD Int. Conf. Manag. Data, 2000, pp. 1-12. 